\title{
REALIZATION OF MAXIMAL SUBGROUPS OF RANK 8 OF THE SIMPLY CONNECTED COMPACT SIMPLE LIE GROUP OF TYPE $E_{8}$
}

\author{
By
}

\author{
Satoshi Gomyo
}

\section{Introduction}

Borel and de Siebenthal ([1]) classified the maximal subgroups of maximal rank of a simply connected compact simple Lie group $G$ and showed that anyone of these subgroups could be realized as the fixed subgroup

$$
G^{\sigma}=\{a \in G \mid \sigma(a)=a\}
$$

of a certain automorphism $\sigma$ of order $p(p=2,3,5)$. The problem of realizing explicitly these automorphisms $\sigma$ and subgroups $G^{\sigma}$ is important. In case $G$ is of classical type, this problem is very easy. In [10], [11] and [12], Yokota and some members of his school realized all $\sigma$ and $G^{\sigma}$ explicitly in cases $G$ were of type $G_{2}$, $F_{4}, E_{6}$ and $E_{7}$. In case $G$ is of type $E_{8}$, this problem has not been solved completely. In this case, it is known ([1]] that the type of these subgroups $G^{\sigma}$ and the order of $\sigma$ are as follows:

$$
\begin{array}{ccccccc}
\text { type of } G^{\sigma} & : & A_{1} \times E_{7} & D_{8} & A_{8} & A_{4} \times A_{4} & A_{2} \times E_{6} \\
\text { order of } \sigma & : & 2 & 2 & 3 & 3 & 5
\end{array}
$$

The subgroups of type $A_{1} \times E_{7}$ and $D_{8}$ have already been realized explicitly in [3], [9] and [10]. One the other hand, Wolf and Gray ([8]) classified the automorphisms of order 3 of the simply connected compact simple Lie group of type $E_{8}$ and showed that the subgroups of type $A_{8}$ and $A_{2} \times E_{6}$ were isomorphic to $S U(9) / Z_{3}$ and $\left(S U(3) \times E_{6}\right) / Z_{3}$, respectively. But the isomorphisms were not completely obtained. In this paper, we shall explicitly give two automorphisms of order 3 such that their fixed subgroups are isomorphic to $S U(9) / Z_{3}$ and $\left(S U(3) \times E_{6}\right) / Z_{3}$ respectively, and an automorphism of order 5 whose fixed 
subgroup is of type $A_{4} \times A_{4}$. The last fixed subgroup is realized as $(S U(5) \times S U(5)) / Z_{5}$.

Finally we remark that three new realizations of the complex simple Lie algebra of type $E_{8}$ are obtained in this paper.

The author wishes to express his sincere thanks to Professor Ichiro Yokota, who motivated the author to study on this subject and to Professor Hiroshi Asano for his valuable suggestions and constant encouragement.

\section{§1. Preliminaries}

1.1 Let $e_{1}, \ldots, e_{n}$ be the canonical basis of $\boldsymbol{C}^{n}$ and $(\boldsymbol{x}, \boldsymbol{y})$ the symmetric bilinear inner product in $C^{n}$ defined by $\left(e_{i}, e_{j}\right)=\delta_{i j}$, where $\delta_{i j}$ means Kronecker's delta. Let us define a bilinear symmetric inner product in the $k$-th exterior power $\bigwedge^{k}\left(C^{n}\right)(0 \leq k \leq n)$ by

$$
\begin{aligned}
\left(x_{1} \wedge \cdots \wedge x_{k}, y_{1} \wedge \cdots \wedge y_{k}\right) & =\operatorname{det}\left(\left(x_{i}, y_{j}\right)\right), & & k \geq 1, \\
(a, b) & =a b, & & a, b \in \wedge^{0}\left(C^{n}\right)=C .
\end{aligned}
$$

Then $\boldsymbol{e}_{i_{1}} \wedge \cdots \wedge \boldsymbol{e}_{i_{k}}\left(i_{1}<\cdots<i_{k}\right)$ forms an orthonormal basis of $\bigwedge^{k}\left(C^{n}\right)$. For any $\boldsymbol{u} \in \bigwedge^{k}\left(\boldsymbol{C}^{n}\right)$, there exists the unique element $*(\boldsymbol{u}) \in \bigwedge^{n-k}\left(\boldsymbol{C}^{n}\right)$ such that

$$
(*(u), v)=\left(u \wedge v, e_{1} \wedge \cdots \wedge e_{n}\right) \text { for } v \in \bigwedge^{n-k}\left(C^{n}\right) .
$$

Then the linear transformation

$$
*: \bigwedge^{k}\left(\boldsymbol{C}^{n}\right) \rightarrow \bigwedge^{n-k}\left(\boldsymbol{C}^{n}\right)
$$

is bijective and it satisfies the following identity:

$$
*^{2}(\boldsymbol{u})=(-1)^{k(n-k)} \boldsymbol{u} \quad\left(\boldsymbol{u} \in \bigwedge^{k}\left(\boldsymbol{C}^{n}\right)\right) .
$$

Let $\rho$ and $d \rho$ be the representations of the complex special linear group $S L(n, C)$ and its Lie algebra $\operatorname{sl}(n, \boldsymbol{C})$ on $\bigwedge^{k}\left(\boldsymbol{C}^{n}\right)(k \geq 1)$ defined by

$$
\begin{aligned}
\rho(A)\left(x_{1} \wedge \cdots \wedge x_{k}\right) & =A x_{1} \wedge \cdots \wedge A x_{k}, \\
d \rho(X)\left(x_{1} \wedge \cdots \wedge x_{k}\right) & =\sum_{j=1}^{k} x_{1} \wedge \cdots \wedge X x_{j} \wedge \cdots \wedge x_{k},
\end{aligned}
$$

respectively. In particular, define the representations $\rho$ of $S L(n, C)$ and $d \rho$ of $\operatorname{sl}(n, \boldsymbol{C})$ on $\bigwedge^{0}\left(\boldsymbol{C}^{n}\right)=\boldsymbol{C}$ by

$$
\rho(A) 1=1, \quad d \rho(X) 1=0 .
$$


Hereafter we shall omit the notations $\rho$ and $d \rho$. We can easily obtain the following:

Lemma 1.1. For $A \in S L(n, C), X \in \mathfrak{s l}(n, C)$ and $u, v \in \bigwedge^{k}\left(C^{n}\right)$, we have

$$
\begin{aligned}
\left(A \boldsymbol{u},{ }^{t} A^{-1} \boldsymbol{v}\right) & =(\boldsymbol{u}, \boldsymbol{v}), \\
(X \boldsymbol{u}, \boldsymbol{v})+\left(\boldsymbol{u},-{ }^{t} X \boldsymbol{v}\right) & =0, \\
*(A \boldsymbol{u}) & ={ }^{t} A^{-1} *(\boldsymbol{u}), \\
*(X \boldsymbol{u}) & =-{ }^{t} X *(\boldsymbol{u}) .
\end{aligned}
$$

For any $u, v \in \bigwedge^{k}\left(C^{n}\right)(1 \leq k \leq n)$, let us define a linear transformation $\boldsymbol{u} \times \boldsymbol{v}$ on $\boldsymbol{C}^{n}$ by

$$
\boldsymbol{u} \times \boldsymbol{v}: x \mapsto *(v \wedge *(\boldsymbol{u} \wedge x))+(-1)^{n-k} \frac{n-k}{n}(\boldsymbol{u}, \boldsymbol{v}) x \quad\left(x \in C^{n}\right) .
$$

Since $\operatorname{tr}(\boldsymbol{u} \times \boldsymbol{v})=0, \boldsymbol{u} \times \boldsymbol{v}$ can be considered as an element of $\operatorname{sI}(n, C)$ with respect to the canonical basis of $\boldsymbol{C}^{n}$. Let - denote the complex conjugation of $\wedge^{k}\left(\boldsymbol{C}^{n}\right)$ with respect to the real form $\bigwedge^{k}\left(\boldsymbol{R}^{n}\right)$ except in the $\S 3$. Furthermore we can easily obtain the following:

Lemma 1.2. For $A \in S L(n, C), X \in \mathfrak{s l}(n, C)$ and $u, v \in \bigwedge^{k}\left(C^{n}\right)$, we have

$$
\begin{aligned}
A(\boldsymbol{u} \times \boldsymbol{v}) A^{-1} & =(A \boldsymbol{u}) \times\left({ }^{t} A^{-1} \boldsymbol{v}\right) \\
{[X, \boldsymbol{u} \times \boldsymbol{v}] } & =(X \boldsymbol{u}) \times \boldsymbol{v}+\boldsymbol{u} \times\left(-{ }^{t} X \boldsymbol{v}\right) \\
t(\boldsymbol{u} \times \boldsymbol{v}) & =\boldsymbol{v} \times \boldsymbol{u} \\
\overline{\boldsymbol{u} \times \boldsymbol{v}} & =\overline{\boldsymbol{u}} \times \overline{\boldsymbol{v}} \\
\operatorname{tr}\{X(\boldsymbol{u} \times \boldsymbol{v})\} & =(-1)^{n-k}(X \boldsymbol{u}, \boldsymbol{v}) .
\end{aligned}
$$

1.2 Let $\mathfrak{g}$ be a complex simple Lie algebra of type $E_{8}$. Since $\mathfrak{g}$ is simple, the Lie algebra $\operatorname{Der}(\mathfrak{g})$ of all derivations of $\mathfrak{g}$ consists of $\operatorname{ad}(R)(R \in \mathfrak{g})$ and it is isomorphic to the Lie algebra $\mathfrak{g}$. Let $\operatorname{Aut}(\mathfrak{g})$ be an automorphism group of $\mathfrak{g}$ and $\operatorname{Innaut}(\mathrm{g})$ an inner automorphism group generated by $\{\exp (\operatorname{ad} R) \mid R \in \mathfrak{g}\}$. Since $\mathfrak{g}$ is of type $E_{8}$, the group $\operatorname{Aut}(\mathfrak{g})$ coincide the group Innaut $(\mathfrak{g})$. Hence $\operatorname{Aut}(\mathfrak{g})$ is connected. Let $\mathfrak{g}^{\prime}$ be a compact real form of $\mathfrak{g}$ and $\gamma$ a conjugation of $\mathfrak{g}$ with respect to $\mathfrak{g}^{\prime}$. Define an inner product on $\mathfrak{g}$ by

$$
\left\langle R_{1}, R_{2}\right\rangle=-B_{\mathfrak{g}}\left(R_{1}, \gamma R_{2}\right) .
$$


Then it is positive definite Hermitian inner product. Let us define a group $E_{8}$ as follows:

$$
E_{8}=\left\{\alpha \in \operatorname{Aut}(\mathfrak{g}) \mid\left\langle\alpha R_{1}, \alpha R_{2}\right\rangle=\left\langle R_{1}, R_{2}\right\rangle\right\} .
$$

Since this group is a closed subgroup of the unitary group

$$
U(\mathfrak{g})=\left\{\alpha \in \operatorname{Iso}(\mathfrak{g}) \mid\left\langle\alpha R_{1}, \alpha R_{2}\right\rangle=\left\langle R_{1}, R_{2}\right\rangle\right\}
$$

the group $E_{8}$ is compact. It is clear that the Lie algebra of this group is isomorphic to $\mathrm{g}^{\prime}$. Hence the group $E_{8}$ is of type $E_{8}$. In order to prove that the group $E_{8}$ is connected, we use the following:

LEMMA 1.3. ([2] p. 450). Let $G$ be an algebraic subgroup of the general linear group $G L(n, C)$ such that the condition $A \in G$ implies $A^{*} \in G$. Then $G$ is homeomorphic to the topological product of $G \cap U(n)$ and a Euclidean space $\boldsymbol{R}^{d}$ :

$$
G \simeq(G \cap U(n)) \times R^{d}
$$

where $U(n)$ is the unitary subgroup of $G L(n, C)$.

It is clear that the group Aut $(\mathfrak{g})$ is the algebraic subgroup of $G L(248, C)=$ Iso $(\mathfrak{g})$. Since $\operatorname{Aut}(\mathfrak{g})$ is generated by $\{\exp (\operatorname{ad} R) \mid R \in \mathfrak{g}\}$ and

$$
\begin{aligned}
\left\langle\exp (\operatorname{ad} R) R_{1}, R_{2}\right\rangle & =-B_{\mathrm{g}}\left(\exp (\operatorname{ad} R) R_{1}, \gamma R_{2}\right) \\
& =B_{\mathrm{g}}\left(R_{1}, \exp (\operatorname{ad} R) \gamma R_{2}\right) \\
& =-B_{\mathrm{g}}\left(R_{1}, \gamma \exp (\operatorname{ad}(-\gamma R)) R_{2}\right) \\
& =\left\langle R_{1}, \exp (\operatorname{ad}(-\gamma R)) R_{2}\right\rangle,
\end{aligned}
$$

$\alpha \in \operatorname{Aut}(\mathfrak{g})$ implies $\alpha^{*} \in \operatorname{Aut}(\mathfrak{g})$, where $\alpha^{*}$ is the transpose of $\alpha$ with respect to $\left\langle R_{1}, R_{2}\right\rangle:\left\langle\alpha R_{1}, R_{2}\right\rangle=\left\langle R_{1}, \alpha^{*} R_{2}\right\rangle$. It is clear that $\operatorname{Aut}(\mathrm{g}) \cap U(\mathrm{~g})=E_{8}$ and $\operatorname{dim}_{R} E_{8}^{C}-\operatorname{dim}_{R} E_{8}=248$. Hence we have

$$
\operatorname{Aut}(\mathfrak{g}) \simeq E_{8} \times R^{248}
$$

Since $\operatorname{Aut}(\mathfrak{g})$ is connected, the group $E_{8}$ is also connected. From the general theory of Lie groups ([7]), the connected compact simple Lie group of type $E_{8}$ is simply connected. Hence we have

Proposition 1.4. Let $\mathfrak{g}$ be a complex simple Lie algebra of type $E_{8}$ and $\gamma$ a conjugation of $\mathfrak{g}$ with respect to a compact real form of $\mathfrak{g}$. Then the group 
$E_{8}=\left\{\alpha \in \operatorname{Aut}(\mathfrak{g}) \mid\left\langle\alpha R_{1}, \alpha R_{2}\right\rangle=\left\langle R_{1}, R_{2}\right\rangle\right\}$ is simply connected compact Lie group of type $E_{8}$.

\section{§2. The subgroup of type $A_{8}$}

In this section, let us consider a complex vector space direct sum

$$
\mathfrak{g}=\mathfrak{s l}(9, C) \oplus \bigwedge^{3}\left(C^{9}\right) \oplus \bigwedge^{3}\left(C^{9}\right)
$$

We define an anti-symmetric bilinear product on $\mathfrak{g}$ by

$$
(X, \boldsymbol{u}, \boldsymbol{v})=\left[\left(X_{1}, \boldsymbol{u}_{1}, \boldsymbol{v}_{1}\right),\left(X_{2}, \boldsymbol{u}_{2}, \boldsymbol{v}_{2}\right)\right]
$$

where

$$
\left\{\begin{array}{l}
X=\left[X_{1}, X_{2}\right]+u_{1} \times v_{2}-u_{2} \times v_{1} \\
u=X_{1} u_{2}-X_{2} u_{1}+*\left(v_{1} \wedge v_{2}\right) \\
v=-{ }^{t} X_{1} v_{2}+{ }^{t} X_{2} v_{1}-*\left(u_{1} \wedge u_{2}\right)
\end{array}\right.
$$

In order to prove the Jacobi identity, we show the following:

LEMMA 2.1. For $\boldsymbol{u}, \boldsymbol{v}, \boldsymbol{w} \in \bigwedge^{3}\left(\boldsymbol{C}^{9}\right)$, we have

$$
\begin{aligned}
& u \times *(v \wedge w)+v \times *(w \wedge u)+w \times *(u \wedge v)=0 \\
& (u \times w) v-(v \times w) u+*(*(u \wedge v) \wedge w)=0
\end{aligned}
$$

Proof. Put $u=u_{1} \wedge u_{2} \wedge u_{3}, v=u_{4} \wedge u_{5} \wedge u_{6}$ and $\boldsymbol{w}=u_{7} \wedge u_{8} \wedge u_{9}$. For $\boldsymbol{x}, \boldsymbol{y} \in \boldsymbol{C}^{9}$, we see

$$
\begin{aligned}
((\boldsymbol{u} \times \boldsymbol{v}) \boldsymbol{x}, \boldsymbol{y})= & (*(\boldsymbol{v} \wedge *(\boldsymbol{u} \wedge \boldsymbol{x})), y)+2 / 3(\boldsymbol{u}, \boldsymbol{v})(\boldsymbol{x}, \boldsymbol{y}) \\
= & -(\boldsymbol{x} \wedge \boldsymbol{u}, \boldsymbol{y} \wedge v)+2 / 3(\boldsymbol{u}, \boldsymbol{v})(\boldsymbol{x}, \boldsymbol{y}) \\
= & \left(x \wedge u_{2} \wedge u_{3}, v\right)\left(u_{1}, y\right)-\left(x \wedge u_{1} \wedge u_{3}, v\right)\left(u_{2}, y\right) \\
& +\left(x \wedge u_{1} \wedge u_{2}, v\right)\left(u_{3}, y\right)-1 / 3(u, v)(x, y) .
\end{aligned}
$$

Hence we have

$$
\begin{aligned}
(u \times v) x= & \left(x \wedge u_{2} \wedge u_{3}, v\right) u_{1}+\left(u_{1} \wedge x \wedge u_{3}, v\right) u_{2} \\
& +\left(u_{1} \wedge u_{2} \wedge x, v\right) u_{3}-1 / 3(u, v) x
\end{aligned}
$$


Using this identity, we have

$$
\begin{aligned}
\{\boldsymbol{u} & \times *(\boldsymbol{v} \wedge \boldsymbol{w})+v \times *(\boldsymbol{w} \wedge \boldsymbol{u})+\boldsymbol{w} \times *(\boldsymbol{u} \wedge v)\} \boldsymbol{x} \\
= & \sum_{j=1}^{9}\left(u_{1} \wedge \cdots \wedge u_{j-1} \wedge x \wedge u_{j+1} \wedge \cdots \wedge u_{9}, e_{1} \wedge \cdots \wedge e_{9}\right) u_{j} \\
& -\left(u_{1} \wedge \cdots \wedge u_{9}, e_{1} \wedge \cdots \wedge e_{9}\right) x=(i) .
\end{aligned}
$$

Let us put $\boldsymbol{x}=\sum_{i=1}^{9} x_{i} \boldsymbol{e}_{i}, u_{j}=\sum_{k=1}^{9} u_{j k} \boldsymbol{e}_{k}$ and $U=\left(u_{j k}\right) \in M(9, C)$. Hence we see

$$
\begin{aligned}
& \left(u_{1} \wedge \cdots \wedge u_{j-1} \wedge x \wedge u_{j+1} \wedge \cdots \wedge u_{9}, e_{1} \wedge \cdots \wedge e_{9}\right)=\sum_{k=1}^{9} \tilde{U}_{j k} x_{k} \\
& \left(u_{1} \wedge \cdots \wedge u_{9}, e_{1} \wedge \cdots \wedge e_{9}\right)=\operatorname{det} U
\end{aligned}
$$

where $\tilde{U}_{j k}$ is the factor of $u_{j k}$ of the matrix $U$. Therefore we have

$$
\begin{aligned}
(\mathrm{i}) & =\sum_{j, k} x_{k} \tilde{U}_{j k} \boldsymbol{u}_{j}-(\operatorname{det} U) \boldsymbol{x}=\sum_{i, j, k} x_{k} \tilde{U}_{j k} u_{j i} \boldsymbol{e}_{i}-(\operatorname{det} U) \boldsymbol{x} \\
& =\sum_{i, k} x_{k}(\operatorname{det} U) \delta_{k i} \boldsymbol{e}_{i}-(\operatorname{det} U) \boldsymbol{x}=0 .
\end{aligned}
$$

Then (1) has been proved. Now let us put $u=u_{1} \wedge u_{2} \wedge u_{3}$ and $v=v_{1} \wedge v_{2} \wedge v_{3}$. Using (3), for any $a \in \bigwedge^{3}\left(C^{9}\right)$ we have

$$
\begin{aligned}
&((u \times w) v-(v \times w) u, a) \\
&=\left(\left((u \times w) v_{1}\right) \wedge v_{2} \wedge v_{3}, a\right)-\left(\left((u \times w) v_{2}\right) \wedge v_{1} \wedge v_{3}, a\right) \\
&+\left(\left((u \times w) v_{3}\right) \wedge v_{1} \wedge v_{2}, a-\left(\left((v \times w) u_{1}\right) \wedge u_{2} \wedge u_{3}, a\right)\right. \\
&+\left(\left((v \times w) u_{2}\right) \wedge u_{1} \wedge u_{3}, a\right)-\left(\left((v \times w) u_{3}\right) \wedge u_{1} \wedge u_{2}, a\right) \\
&=-(u, w)(v, a)+\sum_{i=1}^{3} \sum_{j=1}^{3}\left(u_{i} \wedge u_{i+1} \wedge v_{j}, w\right)\left(u_{i+2} \wedge v_{i+1} \wedge v_{j+2}, a\right) \\
&+(v, w)(u, a)-\sum_{i=1}^{3} \sum_{j=1}^{3}\left(u_{i} \wedge v_{j} \wedge v_{j+1}, w\right)\left(u_{i+1} \wedge u_{i+2} \wedge v_{j+2}, a\right) \\
&=-(u \wedge v, w \wedge a)=-(*(*(u \wedge v) \wedge w), a)
\end{aligned}
$$

Then (2) has been proved.

From Lemmas 1.1, 1.2 and 2.1, we can prove that $g$ becomes a Lie algebra. Furthermore we have the following:

THEOREM 2.2. The Lie algebra $\mathfrak{g}$ is a complex simple Lie algebra of type $E_{8}$. 
Proof. For a subset $I=\{i, j, k\}(i<j<k)$ of $\{1,2, \ldots, 9\}$, we put

$$
\boldsymbol{e}_{I}=\boldsymbol{e}_{i} \wedge \boldsymbol{e}_{j} \wedge \boldsymbol{e}_{k} \in \bigwedge^{3}\left(\boldsymbol{C}^{9}\right)
$$

Let $\mathfrak{a}$ be a non-zero ideal of $\mathfrak{g}$ and let us put

$$
\mathfrak{q}=\bigwedge^{3}\left(\boldsymbol{C}^{9}\right) \oplus \bigwedge^{3}\left(\boldsymbol{C}^{9}\right)
$$

There are three cases to be considered: $(a) \mathfrak{s l}(9, C) \cap \mathfrak{a}=\{0\}$ and $\mathfrak{q} \cap \mathfrak{a}=\{0\}$, (b) $\mathfrak{s l}(9, C) \cap \mathfrak{a} \neq\{0\},(c) \mathfrak{q} \cap \mathfrak{a} \neq\{0\}$.

Case (a): Let $p: \mathfrak{g} \rightarrow \mathfrak{s l}(9, C)$ denote the projection. If $p(\mathfrak{a})=0$, then $\mathfrak{a}$ is contained in $\mathfrak{q}$, which contradicts to $\mathfrak{q} \cap \mathfrak{a}=\{0\}$. For this reason $p(\mathfrak{a})$ is a nonzero ideal of $\mathfrak{s l}(9, \boldsymbol{C})$, hence we have $p(\mathfrak{a})=\mathfrak{s l}(9, C)$. For an element $X=$ $\sum_{i=1}^{8} E_{i i}-8 E_{99} \in \mathfrak{s l}(9, C)$, there exists an element $(\boldsymbol{u}, \boldsymbol{v})=\left(\sum_{I} u_{I} \boldsymbol{e}_{I}, \sum_{J} v_{J} \boldsymbol{e}_{J}\right) \in \mathfrak{q}$ such that $(X, \boldsymbol{u}, \boldsymbol{v}) \in \mathfrak{a}$. Since $[(X, 0,0),(X, \boldsymbol{u}, \boldsymbol{v})]=\left(0, X \boldsymbol{u},-{ }^{t} X \boldsymbol{v}\right) \in \mathfrak{q} \cap \mathfrak{a}=\{0\}$, we have

$$
\begin{aligned}
& 0=X u=\sum_{I} u_{I} X e_{I}=3 \sum_{I \not 99} u_{I} e_{I}-6 \sum_{I \ni 9} u_{I} e_{I}, \\
& 0=-{ }^{t} X v=-3 \sum_{J \not 99} v_{J} e_{J}+6 \sum_{J \ni 9} v_{J} e_{J},
\end{aligned}
$$

i.e., $u_{I}=0$ and $v_{J}=0$. Then $0 \neq(X, \boldsymbol{u}, \boldsymbol{v})=(X, 0,0) \in \mathfrak{s l}(9, \boldsymbol{C}) \cap \mathfrak{a}=\{0\}$. This is a contradiction.

Case (b): Since $\mathfrak{s l}(9, C) \cap \mathfrak{a}$ is a non-zero ideal of $\mathfrak{s l}(9, \boldsymbol{C})$, we have $\mathfrak{s l}(9, C) \subset \mathfrak{a}$. For any $e_{i} \wedge e_{j} \wedge e_{k} \in \bigwedge^{3}\left(C^{9}\right)$, put

$$
X=\frac{1}{3}\left(E_{i i}+E_{j j}+E_{k k}\right)-E_{l l} .
$$

Since $(X, 0,0) \in \mathfrak{s l}(9, C) \subset \mathfrak{a}$, we see that

$$
\begin{aligned}
& \left(0, e_{i} \wedge e_{j} \wedge e_{k}, 0\right)=\left[(X, 0,0),\left(0, e_{i} \wedge e_{j} \wedge e_{k}, 0\right)\right] \in \mathfrak{a} \\
& \left(0,0, e_{i} \wedge e_{j} \wedge e_{k}\right)=\left[(X, 0,0),\left(0,0,-e_{i} \wedge e_{j} \wedge e_{k}\right)\right] \in \mathfrak{a}
\end{aligned}
$$

It follows that $\mathfrak{q} \subset \mathfrak{a}$. Hence we have $\mathfrak{a}=\mathfrak{g}$.

Case (c): Let $R=(0, \boldsymbol{u}, \boldsymbol{v})$ be a non-zero element of $\mathfrak{q} \cap \mathfrak{a}$. In case $\boldsymbol{u} \neq 0$, we put $\boldsymbol{u}=\sum_{I} u_{I} \boldsymbol{e}_{I}$. Without loss of generality, we may assume $u_{\{123\}}=1$. Putting $S_{i j}=\left(E_{i i}-E_{j j}, 0,0\right) \in \mathfrak{g}$ and $T=\left(0,0, e_{1} \wedge e_{2} \wedge e_{4}\right) \in \mathfrak{g}$, we have

$$
\begin{aligned}
0 & \neq \operatorname{ad}(T) \operatorname{ad}\left(S_{37}\right) \operatorname{ad}\left(S_{27}\right) \operatorname{ad}\left(S_{17}\right) \operatorname{ad}\left(S_{36}\right) \operatorname{ad}\left(S_{25}\right) \operatorname{ad}\left(S_{14}\right) R \\
& =\left(-E_{34}, 0,0\right) \in \mathfrak{s l}(9, C) \cap \mathfrak{a} .
\end{aligned}
$$


Then we can reduce this case to case (b). In case $v \neq 0$, we can similarly reduce to case (b).

Thus the simplicity of $\mathfrak{g}$ has been proved. On the other hand, since the dimension of $\mathfrak{g}$ is clearly 248 , we see that $\mathfrak{g}$ is a Lie algebra of type $E_{8}$.

Let us define a conjugate linear transformation $\gamma$ and an inner product $\left\langle R_{1}, R_{2}\right\rangle$ on $\mathrm{g}$ as follows:

$$
\begin{aligned}
\gamma(X, \boldsymbol{u}, \boldsymbol{v}) & =\left(-{ }^{t} \bar{X},-\overline{\boldsymbol{v}},-\overline{\boldsymbol{u}}\right), \\
\left\langle R_{1}, R_{2}\right\rangle & =-B_{\mathrm{g}}\left(R_{1}, \gamma R_{2}\right),
\end{aligned}
$$

where $B_{\mathfrak{g}}$ is the Killing form of $\mathfrak{g}$. We shall show that this inner product is positive definite Hermitian. Now, let us consider another symmetric bilinear form defined by

$$
B_{1}\left(\left(X_{1}, u_{1}, v_{1}\right),\left(X_{2}, u_{2}, v_{2}\right)\right)=\operatorname{tr} X_{1} X_{2}+\left(u_{1}, v_{2}\right)+\left(u_{2}, v_{1}\right) .
$$

Using (1.1), Lemmas 1.1 and 1.2, we see that $B_{1}$ is $\mathrm{g}$-invariant. Since $\mathrm{g}$ is simple, there exists some $\alpha \in C$ such that $B_{\mathfrak{g}}=\alpha B_{1}([5])$. For $R=\left(E_{11}-E_{22}, 0,0\right) \in \mathfrak{g}$, we have $B_{1}(R, R)=2$. On the other hand, we have $B_{\mathfrak{g}}(R, R)=120$ by straightforward calculation. It follows that $B_{\mathfrak{g}}=60 B_{1}$. Hence we have

$$
\left\langle R_{1}, R_{2}\right\rangle=60 \operatorname{tr} X_{1}^{t} \bar{X}_{2}+60\left(u_{1}, \bar{u}_{2}\right)+60\left(v_{1}, \bar{v}_{2}\right),
$$

for $R_{i}=\left(X_{i}, u_{i}, v_{i}\right) \in \mathfrak{g}$. It follows that $\left\langle R_{1}, R_{2}\right\rangle$ is a positive definite Hermitian inner product on g. Using Lemma 1.2 (3) and (4), we see that $\gamma$ holds the Lie bracket. Then it is clear that

$$
\begin{aligned}
\mathfrak{g}^{\gamma} & =\{R \in \mathfrak{g} \mid \gamma(R)=R\} \\
& =\left\{(X, \boldsymbol{u},-\overline{\boldsymbol{u}}) \in \mathfrak{g} \mid X \in \mathfrak{s u}(9), \boldsymbol{u} \in \bigwedge^{3}\left(C^{9}\right)\right\}
\end{aligned}
$$

is the compact real form of $\mathfrak{g}$. Furthermore, from Proposition 1.4, we see that the group

$$
E_{8}=\left\{\alpha \in \operatorname{Aut}(\mathfrak{g}) \mid\left\langle\alpha R_{1}, \alpha R_{2}\right\rangle=\left\langle R_{1}, R_{2}\right\rangle\right\}
$$

is a simply connected compact simple Lie group of type $E_{8}$.

Put $\omega=\exp (2 \pi i / 3) \in C$ and define a transformation $w: \mathfrak{g} \rightarrow \mathfrak{g}$ as follows:

$$
w(X, \boldsymbol{u}, \boldsymbol{v})=\left(X, \omega \boldsymbol{u}, \omega^{2} \boldsymbol{v}\right) .
$$

It is clear that $w \in E_{8}$ and $w^{3}=1$. 
Let $\sigma$ denote the inner automorphism of group $G$ induced by $s \in G$, i.e., $\sigma(x)=s x s^{-1}$. We put $G^{s}$ instead of $G^{\sigma}$.

THEOREM 2.3. The subgroup $\left(E_{8}\right)^{w}$ of $E_{8}$ is isomorphic to the group $S U(9) / Z_{3}$.

Proof. We define a map $\varphi: S U(9) \rightarrow\left(E_{8}\right)^{w}$ by

$$
\varphi(A)(X, \boldsymbol{u}, \boldsymbol{v})=\left(\operatorname{Ad}(A) X, A \boldsymbol{u}^{t} A^{-1} \boldsymbol{v}\right) .
$$

For $Y \in \mathfrak{s u}(9)$, we have

$$
\begin{aligned}
\exp (\operatorname{ad}(Y, 0,0))(X, \boldsymbol{u}, \boldsymbol{v}) & =\left(\exp (\operatorname{ad}(Y)) X,(\exp Y) \boldsymbol{u},\left(\exp \left(-{ }^{t} Y\right)\right) \boldsymbol{v}\right) \\
& =\left(\operatorname{Ad}(\exp Y) X,(\exp Y) \boldsymbol{u},{ }^{t}(\exp Y)^{-1} \boldsymbol{v}\right) \\
& =\varphi(\exp Y)(X, \boldsymbol{u}, \boldsymbol{v}) .
\end{aligned}
$$

Hence $\varphi(A)$ is an automorphism of $\mathfrak{g}$. Furthermore, using Lemma 1.1, we have

$$
\left\langle\varphi(A) R_{1}, \varphi(A) R_{2}\right\rangle=\left\langle R_{1}, R_{2}\right\rangle .
$$

Hence we see $\varphi(A) \in E_{8}$. It is clear that $w \varphi(A)=\varphi(A) w$. Thus the map $\varphi$ is welldefined. Obviously $\varphi$ is a homomorphism. We shall show that $\varphi$ is surjective. The Lie algebra of $\left(E_{8}\right)^{w}$ is isomorphic to

$$
\{R \in \mathfrak{g} \mid \gamma R=R, w R=R\}=\{(X, 0,0) \in \mathfrak{g} \mid X \in \mathfrak{s u}(9)\} \cong \mathfrak{s u}(9) .
$$

Thus the differential of $\varphi$ is surjective. Since $\left(E_{8}\right)^{w}$ is connected [[6]), $\varphi$ is surjective.

At last, we shall show that $\operatorname{Ker} \varphi=\left\{I, \omega I, \omega^{2} I\right\}$. Let $A$ be an element of $\operatorname{Ker} \varphi$. Since $\operatorname{Ad}(A) X=X$, we have $A=\zeta^{m} I$ where $m \in Z$ and $\zeta=\exp (2 \pi i / 9) \in$ $\boldsymbol{C}\left(\zeta^{3}=\omega\right)$. Since

$$
(X, \boldsymbol{u}, \boldsymbol{v})=\varphi\left(\zeta^{m} I\right)(X, \boldsymbol{u}, \boldsymbol{v})=\left(X, \zeta^{3 m} \boldsymbol{u}, \zeta^{6 m} \boldsymbol{v}\right)=\left(X, \omega^{m} \boldsymbol{u}, \omega^{2 m} \boldsymbol{v}\right),
$$

we have $m \equiv 0 \bmod 3$. Then we see that $\operatorname{Ker} \varphi=\left\{I, \omega I, \omega^{2} I\right\} \cong Z_{3}$.

Therefore $S U(9) / Z_{3} \cong\left(E_{8}\right)^{w}$ has been proved.

This theorem means that $\left(E_{8}\right)^{w}$ is a subgroup of type $A_{8}$.

\section{§3. The subgroup of type $A_{2} \times E_{6}$}

In this section, we denote the complexification of any real vector space $S$ by $S^{C}$. And the complex conjugation of $S^{C}$ with respect to the real form $S$ is 
denoted by $\tau$ instead of ${ }^{-}$in $\S 1, \S 2$ and $\S 4$, because we have to distinguish it from the following canonical involution - of Cayley algebra. The complex conjugation in $\boldsymbol{C}=\boldsymbol{R}^{\boldsymbol{C}}$ is also denoted by $\tau$.

Let $\mathfrak{C}$ be the division Cayley algebra over $\boldsymbol{R}$. We denote the canonical involution of $\mathfrak{C}$ by $\bar{x}(x \in C)$. Let

$$
\begin{aligned}
& \mathfrak{I}=\left\{U \in M(3, \mathfrak{C}) \mid U={ }^{t} \bar{U}\right\} \\
& \left.=\left\{\begin{array}{lll}
a_{1} & x_{3} & \bar{x}_{2} \\
\bar{x}_{3} & a_{2} & x_{1} \\
x_{2} & \bar{x}_{1} & a_{3}
\end{array}\right) \in M(3, \mathfrak{C}) \mid \begin{array}{l}
a_{i} \subset \boldsymbol{R} \\
x_{i} \in \mathfrak{C}
\end{array}\right\}
\end{aligned}
$$

be the Jordan algebra over $\boldsymbol{R}$ with respect to the Jordan multiplication

$$
U \circ V=\frac{1}{2}(U V+V U) \text {. }
$$

In $\mathfrak{J}^{C}$, a symmetric inner product $(U, V)$, a positive definite Hermitian inner product $\langle U, V\rangle$, a cross product $U \times V$, a cubic form $(U, V, W)$ and the determinant $\operatorname{det} U$ are defined respectively by

$$
\begin{aligned}
(U, V) & =\operatorname{tr}(U \circ V), \quad\langle U, V\rangle=(\tau U, V), \\
U \times V & =U \circ V-\frac{1}{2}(\operatorname{tr}(U) V+\operatorname{tr}(V) U)+\frac{1}{2}\{\operatorname{tr}(U) \operatorname{tr}(V)-(U, V)\} I, \\
(U, V, W) & =(U, V \times W)=(U \times V, W), \quad \operatorname{det} U=(U, U, U),
\end{aligned}
$$

where $I$ means the $3 \times 3$ unit matrix.

In [10], Yokota realized a complex simple Lie algebra $e_{6}^{C}$ of type $E_{6}$ as

$$
\mathrm{e}_{6}^{\mathrm{C}}=\left\{\phi \in \operatorname{Hom}_{C}\left(\mathfrak{J}^{C}, \mathfrak{J}^{C}\right) \mid(\phi U, U, U)=0\right\}
$$

and he showed that the group

$$
E_{6}=\left\{\begin{array}{l|l}
\alpha \in \operatorname{Iso}_{C}\left(\mathfrak{I}^{C}\right) & \begin{array}{l}
\operatorname{det} \alpha U=\operatorname{det} U \\
\langle\alpha U, \alpha V\rangle=\langle U, V\rangle
\end{array}
\end{array}\right\}
$$

is a simply connected compact simple Lie group of type $E_{6}$, whose Lie algebra is

$$
\begin{aligned}
\mathfrak{e}_{6} & =\left\{\phi \in \mathrm{e}_{6}^{C} \mid\langle\phi U, V\rangle+\langle U, \phi V\rangle=0\right\} \\
& =\left\{\phi \in \mathrm{e}_{6}^{C} \mid-\tau^{t} \phi \tau=\phi\right\},
\end{aligned}
$$

where ${ }^{t} \phi$ means the transpose of $\phi$ with respect to $(U, V)$. For $U, V \in \mathfrak{I}^{C}$, define 
$U \vee V \in \mathrm{e}_{6}^{C}$ by

$$
(U \vee V) X=\frac{1}{2}(V, X) U+\frac{1}{6}(U, V) X-2 V \times(U \times X), \quad\left(X \in \mathfrak{J}^{C}\right) .
$$

Now, we consider a complex 81-dimensional vector space $C^{3} \otimes \mathfrak{J}^{C}$. We denote each element of $C^{3} \otimes \mathfrak{J}^{C}$ in matrix form as

$$
\boldsymbol{U}=\left(U_{i}\right)=\left[\begin{array}{l}
U_{1} \\
U_{2} \\
U_{3}
\end{array}\right] \quad\left(U_{i} \in \mathfrak{J}^{C}\right) .
$$

For $\phi \in \operatorname{Hom}\left(\mathfrak{J}^{C}, \mathfrak{J}^{\boldsymbol{C}}\right), X=\left(x_{i j}\right) \in M(3, \boldsymbol{C})$ and $\boldsymbol{U}=\left(U_{i}\right) \in \boldsymbol{C}^{3} \otimes \mathfrak{J}^{\boldsymbol{C}}$, define $\phi \boldsymbol{U}, X \boldsymbol{U} \in \boldsymbol{C}^{3} \otimes \mathfrak{I}^{\boldsymbol{C}}$ as follows:

$$
\phi \boldsymbol{U}=\left[\begin{array}{l}
\phi U_{1} \\
\phi U_{2} \\
\phi U_{3}
\end{array}\right], \quad X \boldsymbol{U}=\left[\begin{array}{l}
x_{11} U_{1}+x_{12} U_{2}+x_{13} U_{3} \\
x_{21} U_{1}+x_{22} U_{2}+x_{23} U_{3} \\
x_{31} U_{1}+x_{32} U_{2}+x_{33} U_{3}
\end{array}\right] .
$$

For $\boldsymbol{U}=\left(U_{i}\right), \boldsymbol{V}=\left(V_{i}\right) \in \boldsymbol{C}^{3} \otimes \mathfrak{J}^{C}$, let us define a symmetric inner product $(\boldsymbol{U}, \boldsymbol{V})$, a positive definite Hermitian inner product $\langle\boldsymbol{U}, \boldsymbol{V}\rangle$, a cross product $\boldsymbol{U} \times \boldsymbol{V}$, an element $\boldsymbol{U} \circ \boldsymbol{V}$ of $\mathfrak{s l}(3, \boldsymbol{C})$ and an element $U \vee V$ of $\mathrm{e}_{6}^{C}$ by

$$
\begin{aligned}
& (\boldsymbol{U}, \boldsymbol{V})=\left(U_{1}, V_{1}\right)+\left(U_{2}, V_{2}\right)+\left(U_{3}, V_{3}\right), \\
& \langle\boldsymbol{U}, \boldsymbol{V}\rangle=\left\langle U_{1}, V_{1}\right\rangle+\left\langle U_{2}, V_{2}\right\rangle+\left\langle U_{3}, V_{3}\right\rangle, \\
& \boldsymbol{U} \times \boldsymbol{V}=\left[\begin{array}{l}
U_{2} \times V_{3}-V_{2} \times U_{3} \\
U_{3} \times V_{1}-V_{3} \times U_{1} \\
U_{1} \times V_{2}-V_{1} \times U_{2}
\end{array}\right], \\
& \boldsymbol{U} \circ \boldsymbol{V}=\left[\begin{array}{lll}
\left(U_{1}, V_{1}\right) & \left(U_{1}, V_{2}\right) & \left(U_{1}, V_{3}\right) \\
\left(U_{2}, V_{1}\right) & \left(U_{2}, V_{2}\right) & \left(U_{2}, V_{3}\right) \\
\left(U_{3}, V_{1}\right) & \left(U_{3}, V_{2}\right) & \left(U_{3}, V_{3}\right)
\end{array}\right]-\frac{1}{3}(\boldsymbol{U}, \boldsymbol{V}) I, \\
& \boldsymbol{U} \vee \boldsymbol{V}=U_{1} \vee V_{1}+U_{2} \vee V_{2}+U_{3} \vee V_{3},
\end{aligned}
$$

respectively.

Next, let us consider a complex vector space direct sum

$$
\mathrm{m}=\mathfrak{s l}(3, C) \oplus \mathrm{e}_{6}^{C} \oplus C^{3} \otimes \mathfrak{J}^{C} \oplus C^{3} \otimes \mathfrak{J}^{C} .
$$

Furthermore, let us define an anti-symmetric bilinear product on $m$ as follows:

$$
(X, \phi, U, V)=\left[\left(X_{1}, \phi_{1}, U_{1}, V_{1}\right),\left(X_{2}, \phi_{2}, U_{2}, V_{2}\right)\right]
$$


where

$$
\left\{\begin{array}{l}
X=\left[X_{1}, X_{2}\right]+\frac{1}{4} U_{1} \circ V_{2}-\frac{1}{4} U_{2} \circ V_{1} \\
\phi=\left[\phi_{1}, \phi_{2}\right]+\frac{1}{2} U_{1} \vee V_{2}-\frac{1}{2} U_{2} \vee V_{1} \\
U=\phi_{1} U_{2}-\phi_{2} U_{1}+X_{1} U_{2}-X_{2} U_{1}-V_{1} \times V_{2} \\
V=-{ }^{t} \phi_{1} V_{2}+{ }^{t} \phi_{2} V_{1}-{ }^{t} X_{1} V_{2}+{ }^{t} X_{2} V_{1}+U_{1} \times U_{2}
\end{array}\right.
$$

Let $\mathrm{e}_{8}^{C}$ be a complex simple Lie algebra of type $E_{8}$ which was realized in [4];

$$
\mathrm{e}_{8}^{C}=\mathrm{e}_{7}^{C} \oplus \mathfrak{P}^{C} \oplus \mathfrak{P}^{C} \oplus \boldsymbol{C} \oplus \boldsymbol{C} \oplus \boldsymbol{C} .
$$

Define a map $\mu: \mathrm{e}_{8}^{C} \rightarrow \mathrm{m}$ as follows:

$$
\begin{aligned}
& \mu(\Phi(\phi, S, T, v),(U, V, \xi, \eta),(W, Y, \zeta, \omega), r, s, t) \\
& =\left(\left[\begin{array}{ccc}
\frac{2}{3} v & -\frac{1}{2} \xi & \frac{1}{2} \zeta \\
\frac{1}{2} \omega & -\frac{1}{3} v-r & t \\
\frac{1}{2} \eta & s & -\frac{1}{3} v+r
\end{array}\right], \phi,\left[\begin{array}{c}
-2 S \\
W \\
U
\end{array}\right],\left[\begin{array}{c}
-2 T \\
V \\
-Y
\end{array}\right]\right) .
\end{aligned}
$$

We can prove that $\mu$ is isomorphism by straightforward calculation. Thus we have

THEOREM 3.1. The Lie algebra $\mathfrak{m}$ is a complex simple Lie algebra of type $E_{8}$.

Using the Killing form of $e_{8}^{C}$ which was obtained in [4], we see that the Killing form $B_{\mathfrak{m}}$ is

$$
B_{\mathfrak{m}}\left(R_{1}, R_{2}\right)=60 \operatorname{tr}\left(X_{1} X_{2}\right)+\frac{5}{2} B_{\mathrm{e}_{6}^{c}}\left(\phi_{1}, \phi_{2}\right)+15\left(U_{1}, V_{2}\right)+15\left(U_{2}, V_{1}\right),
$$

for $R_{i}=\left(X_{i}, \phi_{i}, U_{i}, V_{i}\right) \in \mathrm{m}$. We define a conjugate linear transformation $\gamma$ and an inner product $\left\langle R_{1}, R_{2}\right\rangle$ on $\mathrm{m}$ as follows:

$$
\begin{aligned}
\gamma(\boldsymbol{X}, \boldsymbol{\phi}, \boldsymbol{U}, \boldsymbol{V}) & =\left(-\tau^{t} \boldsymbol{X},-\tau^{t} \phi \tau,-\tau \boldsymbol{V},-\tau \boldsymbol{U}\right) \\
\left\langle\boldsymbol{R}_{1}, \boldsymbol{R}_{2}\right\rangle & =-\boldsymbol{B}_{\mathfrak{m}}\left(\boldsymbol{R}_{1}, \gamma \boldsymbol{R}_{2}\right) .
\end{aligned}
$$

Then we have

$$
\left\langle R_{1}, R_{2}\right\rangle=60 \operatorname{tr} X_{1}\left(\tau^{t} X_{2}\right)+\frac{5}{2} B_{\mathrm{e}_{6}^{c}}\left(\phi_{1}, \tau^{t} \phi_{2} \tau\right)+15\left\langle U_{1}, U_{2}\right\rangle+15\left\langle V_{1}, V_{2}\right\rangle
$$


This implies that $\left\langle R_{1}, R_{2}\right\rangle$ is a positive definite Hermitian inner product on $\mathrm{m}$. Using the following equations:

$$
\tau^{t}(\boldsymbol{U} \circ \boldsymbol{V})=(\tau \boldsymbol{V}) \circ(\tau \boldsymbol{U}) \quad \text { and } \quad \tau^{t}(\boldsymbol{U} \vee \boldsymbol{V})=(\tau \boldsymbol{V}) \vee(\tau \boldsymbol{U})
$$

we see that $\gamma$ holds the Lie bracket. Then it is clear that $\mathrm{m}^{\gamma}=\{R \in \mathfrak{m} \mid \gamma(R)=R\}$ is the compact real form of $m$. Furthermore, from Proposition 1.4, we see that the group

$$
E_{8}=\left\{\alpha \in \operatorname{Aut}(\mathrm{m}) \mid\left\langle\alpha R_{1}, \alpha R_{2}\right\rangle=\left\langle R_{1}, R_{2}\right\rangle\right\}
$$

is a simply connected compact simple Lie group of type $E_{8}$.

Let us define a transformation $\delta: m \rightarrow m$ as follows:

$$
\delta(X, \phi, \boldsymbol{U}, \boldsymbol{V})=\left(X, \phi, \omega \boldsymbol{U}, \omega^{2} \boldsymbol{V}\right)
$$

It is clear that $\delta \in E_{8}$ and $\delta^{3}=1$.

THEOREM 3.2. The subgroup $\left(E_{8}\right)^{\delta}$ is isomorphic to the group $\left(S U(3) \times E_{6}\right) / Z_{3}$

Proof. For any $A \in S U(3)$, we define a linear transformation $\psi(A)$ on $\mathrm{m}$ by

$$
\psi(A)(X, \phi, U, V)=\left(\operatorname{Ad}(A) X, \phi, A \boldsymbol{U},{ }^{t} A^{-1} \boldsymbol{V}\right) .
$$

Obviously the map $\psi: S U(3) \rightarrow G L(\mathfrak{m})$ is a homomorphism. Furthermore, since

$$
\exp (\operatorname{ad}(Y, 0,0,0))=\psi(\exp Y), \quad(Y \in \mathfrak{s u}(3)),
$$

we see that $\psi(A)$ is an automorphism of $m$. For any $U, V \in C^{3} \otimes \mathfrak{J}^{C}$, we have

$$
\langle A \boldsymbol{U}, A \boldsymbol{V}\rangle=\langle\boldsymbol{U}, \boldsymbol{V}\rangle
$$

It follows that

$$
\left\langle\psi(A) R_{1}, \psi(A) R_{2}\right\rangle=\left\langle R_{1}, R_{2}\right\rangle
$$

Hence $\psi(A) \in E_{8}$. Similarly, for any $\alpha \in E_{6}$, we define a linear transformation $\mu(\alpha)$ on $\mathrm{m}$ by

$$
\mu(\alpha)(X, \phi, \boldsymbol{U}, \boldsymbol{V})=\left(X, \operatorname{Ad}(\alpha) \phi, \alpha \boldsymbol{U},{ }^{t} \alpha^{-1} \boldsymbol{V}\right)
$$

where ${ }^{t} \alpha$ means the transpose of $\alpha$ with respect to $(U, V)$. It is clear that the map 
$\mu: E_{6} \rightarrow G L(m)$ is homomorphism. Since

$$
\alpha U_{1} \times \alpha U_{2}={ }^{t} \alpha^{-1}\left(U_{1} \times U_{2}\right), \quad\left(\alpha U_{1},{ }^{t} \alpha^{-1} u_{2}\right)=\left(U_{1}, U_{2}\right), \quad\left(U_{i} \in \mathfrak{J}^{\boldsymbol{C}}\right),
$$

$\mu(\alpha)$ is an automorphism of $\mathrm{m}$. Since

$$
B_{\mathrm{e}_{6}^{c}}\left(\operatorname{Ad}(\alpha) \phi_{1}, \tau^{t}\left(\operatorname{Ad}(\alpha) \phi_{2}\right) \tau\right)=B_{\mathrm{e}_{6}^{c}}\left(\phi_{1}, \tau^{t} \phi_{2} \tau\right),
$$

we have

$$
\left\langle\mu(\alpha) R_{1}, \mu(\alpha) R_{2}\right\rangle=\left\langle R_{1}, R_{2}\right\rangle .
$$

Hence $\mu(\alpha) \in E_{8}$. It is clear that $\psi(A) \mu(\alpha)=\mu(\alpha) \psi(A)$.

Furthermore we define a map $\varphi: S U(3) \times E_{6} \rightarrow\left(E_{8}\right)^{\delta}$ by

$$
\varphi(A, \alpha)=\psi(A) \mu(\alpha) .
$$

Since $\delta=\psi(\omega I)$, we have $\delta \varphi(A, \alpha)=\varphi(A, \alpha) \delta$. Thus the map $\varphi$ is well-defined. Obviously $\varphi$ is a homomorphism. Also we can prove that $\varphi$ is surjective as in proof of Theorem 2.3.

Next, we shall show that $\left.\operatorname{Ker} \varphi=\left\{(I, 1), \omega I, \omega^{2} 1\right),\left(\omega^{2} I, \omega 1\right)\right\}$. For $(A, \alpha) \in$ $\operatorname{Ker} \varphi$, we have $A=\omega^{m} I$ and $\alpha=\omega^{n} 1$ where $n, m \in Z$. Since

$$
(X, \phi, \boldsymbol{U}, \boldsymbol{V})=\varphi\left(\omega^{m} I, \omega^{n} 1\right)(X, \phi, \boldsymbol{U}, \boldsymbol{V})=\left(X, \phi, \omega^{m+n} \boldsymbol{U}, \omega^{2(m+n)} \boldsymbol{V}\right),
$$

we have that $m+n \equiv 0 \bmod 3$. Thus we have

$$
\operatorname{Ker} \varphi=\left\{(I, 1),\left(\omega I, \omega^{2} 1\right),\left(\omega^{2} I, \omega 1\right)\right\} \cong Z_{3} .
$$

Therefore $\left(S U(3) \times E_{6}\right) / Z_{3} \cong\left(E_{8}\right)^{\delta}$ has been proved.

This theorem means that $\left(E_{8}\right)^{\delta}$ is a subgroup of type $A_{2} \times E_{6}$.

\section{§4. The subgroup of type $A_{4} \times A_{4}$}

In this section, let us consider a complex vector space direct sum

$$
\mathfrak{I}=\mathfrak{I}_{0} \oplus \mathfrak{I}_{1} \oplus \mathfrak{I}_{2} \oplus \mathfrak{I}_{3} \oplus \mathfrak{I}_{4}
$$

where

$$
\begin{array}{ll}
\mathfrak{I}_{0}=\mathfrak{s l}(5, \boldsymbol{C}) \oplus \mathfrak{s l}(5, \boldsymbol{C}), & \\
\mathfrak{I}_{1}=\bigwedge^{1}\left(\boldsymbol{C}^{5}\right) \otimes \bigwedge^{2}\left(\boldsymbol{C}^{5}\right), & \mathfrak{I}_{2}=\bigwedge^{2}\left(\boldsymbol{C}^{5}\right) \otimes \bigwedge^{1}\left(\boldsymbol{C}^{5}\right), \\
\mathfrak{I}_{3}=\bigwedge^{2}\left(\boldsymbol{C}^{5}\right) \otimes \bigwedge^{1}\left(\boldsymbol{C}^{5}\right), & \mathfrak{I}_{4}=\bigwedge^{1}\left(\boldsymbol{C}^{5}\right) \otimes \bigwedge^{2}\left(\boldsymbol{C}^{5}\right) .
\end{array}
$$


We define an anti-symmetric bilinear product on $\mathfrak{l}$ as follows:

$$
\begin{aligned}
& {\left[\mathrm{I}_{0}, \mathrm{I}_{0}\right] \subset \mathrm{I}_{0}, \quad\left[\left(X_{1}, Y_{1}\right),\left(X_{2}, Y_{2}\right)\right]=\left(\left[X_{1}, X_{2}\right],\left[Y_{1}, Y_{2}\right]\right),} \\
& {\left[\mathrm{I}_{0}, \mathrm{I}_{1}\right] \subset \mathrm{I}_{1}, \quad[(X, Y), \boldsymbol{x} \otimes \boldsymbol{a}]=(X \boldsymbol{x}) \otimes \boldsymbol{a}+\boldsymbol{x} \otimes(Y \boldsymbol{a}),} \\
& {\left[\mathfrak{I}_{0}, \mathfrak{I}_{2}\right] \subset \mathfrak{I}_{2}, \quad[(X, Y), \quad \boldsymbol{b} \otimes \boldsymbol{y}]=(X \boldsymbol{b}) \otimes \boldsymbol{y}+\boldsymbol{b} \otimes\left(-{ }^{t} Y \boldsymbol{y}\right),} \\
& {\left[\mathrm{I}_{0}, \mathrm{I}_{3}\right] \subset \mathrm{I}_{3}, \quad[(X, Y), c \otimes z]=\left(-{ }^{t} X \boldsymbol{c}\right) \otimes z+c \otimes(Y z),} \\
& {\left[\mathfrak{I}_{0}, \mathfrak{I}_{4}\right] \subset \mathfrak{I}_{4}, \quad[(X, Y), \boldsymbol{w} \otimes d]=\left(-{ }^{t} X \boldsymbol{w}\right) \otimes d+w \otimes\left(-{ }^{t} Y d\right),} \\
& {\left[\mathfrak{I}_{1}, \mathfrak{I}_{4}\right] \subset \mathfrak{I}_{0}, \quad[\boldsymbol{x} \otimes \boldsymbol{a}, \boldsymbol{w} \otimes \boldsymbol{d}]=(-(\boldsymbol{a}, \boldsymbol{d}) \boldsymbol{x} \times \boldsymbol{w},(\boldsymbol{x}, \boldsymbol{w}) \boldsymbol{a} \times \boldsymbol{d}),} \\
& {\left[\mathfrak{I}_{2}, \mathfrak{I}_{3}\right] \subset \mathfrak{I}_{0}, \quad[\boldsymbol{b} \otimes \boldsymbol{y}, \boldsymbol{c} \otimes \boldsymbol{z}]=((\boldsymbol{y}, \boldsymbol{z}) \boldsymbol{b} \times \boldsymbol{c},(\boldsymbol{b}, \boldsymbol{c}) \boldsymbol{z} \times \boldsymbol{y}),} \\
& {\left[\mathfrak{I}_{1}, \mathfrak{l}_{1}\right] \subset \mathfrak{I}_{2} \text { and }\left[\mathfrak{I}_{4}, \mathfrak{I}_{4}\right] \subset \mathfrak{I}_{3}} \\
& {\left[x_{1} \otimes a_{1}, x_{2} \otimes a_{2}\right]=\left(x_{1} \wedge x_{2}\right) \otimes *\left(a_{1} \wedge a_{2}\right),} \\
& {\left[\mathrm{I}_{2}, \mathrm{I}_{2}\right] \subset \mathrm{I}_{4} \text { and }\left[\mathrm{I}_{3}, \mathrm{I}_{3}\right] \subset \mathrm{I}_{1} \text {, }} \\
& {\left[b_{1} \otimes y_{1}, b_{2} \otimes y_{2}\right]=*\left(b_{1} \wedge b_{2}\right) \otimes\left(y_{1} \wedge y_{2}\right),} \\
& {\left[\mathrm{I}_{1}, \mathrm{I}_{2}\right] \subset \mathfrak{I}_{3} \quad \text { and } \quad\left[\mathrm{I}_{4}, \mathfrak{I}_{3}\right] \subset \mathfrak{I}_{2} \text {, }} \\
& {[\boldsymbol{x} \otimes \boldsymbol{a}, \boldsymbol{b} \otimes \boldsymbol{y}]=*(\boldsymbol{b} \wedge \boldsymbol{x}) \otimes *(*(\boldsymbol{a}) \wedge \boldsymbol{y}),} \\
& {\left[\mathfrak{I}_{2}, \mathfrak{I}_{4}\right] \subset \mathfrak{I}_{1} \text { and }\left[\mathfrak{I}_{3}, \mathfrak{I}_{1}\right] \subset \mathfrak{I}_{4},} \\
& {[\boldsymbol{b} \otimes \boldsymbol{y}, \boldsymbol{w} \otimes \boldsymbol{d}]=*(*(\boldsymbol{b}) \wedge \boldsymbol{w}) \otimes *(\boldsymbol{d} \wedge \boldsymbol{y}) .}
\end{aligned}
$$

In order to prove the Jacobi identity, we show the following:

LEMMA 4.1. For $\boldsymbol{x}, \boldsymbol{y}, z \in \bigwedge^{1}\left(\boldsymbol{C}^{5}\right)\left(=\boldsymbol{C}^{5}\right)$ and $\boldsymbol{a}, \boldsymbol{b}, \boldsymbol{c} \in \bigwedge^{2}\left(\boldsymbol{C}^{5}\right)$, we have

$$
\begin{aligned}
& *(\boldsymbol{a}) \wedge *(\boldsymbol{b} \wedge \boldsymbol{c})+*(\boldsymbol{b}) \wedge *(\boldsymbol{c} \wedge \boldsymbol{a})+*(\boldsymbol{c}) \wedge *(\boldsymbol{a} \wedge \boldsymbol{b})=0 \\
& *(\boldsymbol{a} \wedge *(*(\boldsymbol{b}) \wedge \boldsymbol{x}))+*(\boldsymbol{b} \wedge *(*(\boldsymbol{a}) \wedge \boldsymbol{x}))+\boldsymbol{x} \wedge *(\boldsymbol{a} \wedge \boldsymbol{b})=0 \\
& *(*(\boldsymbol{x} \wedge \boldsymbol{y}) \wedge \boldsymbol{z})=(\boldsymbol{x}, \boldsymbol{z}) \boldsymbol{y}-(\boldsymbol{y}, \boldsymbol{z}) \boldsymbol{x} \\
& \boldsymbol{x} \wedge *(*(\boldsymbol{a}) \wedge \boldsymbol{y})+*(\boldsymbol{y} \wedge *(\boldsymbol{a} \wedge \boldsymbol{x}))-(\boldsymbol{x}, \boldsymbol{y}) \boldsymbol{a}=0 \\
& *(\boldsymbol{a} \wedge *(\boldsymbol{b} \wedge \boldsymbol{x}))-*(*(\boldsymbol{b}) \wedge *(*(\boldsymbol{a}) \wedge \boldsymbol{x}))-(\boldsymbol{a}, \boldsymbol{b}) \boldsymbol{x}=0 \\
& \boldsymbol{a} \times *(\boldsymbol{b} \wedge \boldsymbol{x})+\boldsymbol{b} \times *(\boldsymbol{a} \wedge \boldsymbol{x})-\boldsymbol{x} \times *(\boldsymbol{a} \wedge \boldsymbol{b})=0 \\
& *(*(\boldsymbol{a}) \wedge \boldsymbol{x}) \times \boldsymbol{y}-*(*(\boldsymbol{a}) \wedge \boldsymbol{y}) \times \boldsymbol{x}+\boldsymbol{a} \times(\boldsymbol{x} \wedge \boldsymbol{y})=0 \\
& (\boldsymbol{a} \times \boldsymbol{b}) \boldsymbol{c}=*(*(\boldsymbol{a} \wedge \boldsymbol{c}) \wedge \boldsymbol{b})-1 / 5(\boldsymbol{a}, \boldsymbol{b}) \boldsymbol{c}-(\boldsymbol{b}, \boldsymbol{c}) \boldsymbol{a} \\
& (\boldsymbol{x} \times \boldsymbol{y}) \boldsymbol{a}=-*(\boldsymbol{y} \wedge *(\boldsymbol{x} \wedge \boldsymbol{a}))+3 / 5(\boldsymbol{x}, \boldsymbol{y}) \boldsymbol{a} .
\end{aligned}
$$


Proof. (1): Let us put $a=a_{1} \wedge a_{2}, \quad b=a_{3} \wedge a_{4}, c=a_{5} \wedge a_{6}$ and $a_{i}=$ $\sum_{j=1}^{5} a_{i j} \boldsymbol{e}_{j}$. Since

$$
\begin{aligned}
(*(*(a) \wedge *(b \wedge c)), x) & =(a, *(b \wedge c) \wedge x) \\
& =\left(a_{1}, *(b \wedge c)\right)\left(a_{2}, x\right)-\left(a_{2}, *(b \wedge c)\right)\left(a_{1}, x\right) \\
& =\left(a_{1} \wedge b \wedge c, e_{1} \wedge \cdots \wedge e_{5}\right)\left(a_{2}, x\right) \\
& -\left(a_{2} \wedge b \wedge c, e_{1} \wedge \cdots \wedge e_{5}\right)\left(a_{1}, x\right)
\end{aligned}
$$

we have

$$
\begin{aligned}
& *(*(\boldsymbol{a}) \wedge *(\boldsymbol{b} \wedge \boldsymbol{c})+*(\boldsymbol{b}) \wedge *(\boldsymbol{c} \wedge \boldsymbol{a})+*(\boldsymbol{c}) \wedge *(\boldsymbol{a} \wedge \boldsymbol{b})) \\
& =\sum_{j=1}^{5} \sum_{i=1}^{6}(-1)^{i}\left(\boldsymbol{a}_{1} \wedge \cdots \wedge \boldsymbol{a}_{i-1} \wedge \boldsymbol{a}_{i+1} \wedge \cdots \wedge a_{6}, \boldsymbol{e}_{1} \wedge \cdots \wedge \boldsymbol{e}_{5}\right) a_{i j} \boldsymbol{e}_{j} \\
& =-\sum_{j=1}^{5} \operatorname{det}\left[\begin{array}{cccc}
a_{1 j} & a_{11} & \cdots & a_{15} \\
a_{2 j} & a_{21} & \cdots & a_{25} \\
\vdots & \vdots & \ddots & \vdots \\
a_{6 j} & a_{61} & \cdots & a_{65}
\end{array}\right] \boldsymbol{e}_{j}=0 .
\end{aligned}
$$

(2): Using (1), we have

$$
\begin{aligned}
& (*(\boldsymbol{a} \wedge *(*(\boldsymbol{b}) \wedge \boldsymbol{x}))+*(\boldsymbol{b} \wedge *(*(\boldsymbol{a}) \wedge \boldsymbol{x}))+\boldsymbol{x} \wedge *(\boldsymbol{a} \wedge \boldsymbol{b}), \boldsymbol{c}) \\
& =(*(\boldsymbol{b}) \wedge \boldsymbol{x}, \boldsymbol{c} \wedge \boldsymbol{a})+(*(\boldsymbol{a}) \wedge \boldsymbol{x}, \boldsymbol{b} \wedge \boldsymbol{c})+(*(\boldsymbol{c}) \wedge \boldsymbol{x}, \boldsymbol{a} \wedge \boldsymbol{b}) \\
& =(*(\boldsymbol{x}), *(\boldsymbol{b}) \wedge *(\boldsymbol{c} \wedge \boldsymbol{a})+*(\boldsymbol{a}) \wedge *(\boldsymbol{b} \wedge \boldsymbol{c})+*(\boldsymbol{c}) \wedge *(\boldsymbol{a} \wedge \boldsymbol{b}))=0 .
\end{aligned}
$$

(3): For any $v \in \bigwedge^{1}\left(C^{5}\right)=C^{5}$, we have

$$
(*(*(x \wedge y) \wedge z), v)=(x \wedge y, z \wedge v)=(x, z)(y, v)-(y, z)(x, v) .
$$

Then (3) has proved. (4) and (5): Let us put $a=a_{1} \wedge a_{2}$. Since

$$
\begin{aligned}
(x \wedge a, y \wedge b) & =(x, y)(a, b)-\left(a_{1}, y\right)\left(x \wedge a_{2}, b\right)+\left(a_{2}, y\right)\left(x \wedge a_{1}, b\right) \\
(*(b) \wedge x, *(a) \wedge y) & =(a, y \wedge *(*(b) \wedge x)) \\
& =\left(a_{1}, y\right)\left(x \wedge a_{2}, b\right)-\left(a_{2}, y\right)\left(x \wedge a_{1}, b\right)
\end{aligned}
$$

we have

$$
(\boldsymbol{x} \wedge \boldsymbol{a}, \boldsymbol{y} \wedge \boldsymbol{b})+(*(\boldsymbol{b}) \wedge \boldsymbol{x}, *(\boldsymbol{a}) \wedge \boldsymbol{y})=(\boldsymbol{x}, \boldsymbol{y})(\boldsymbol{a}, \boldsymbol{b}) .
$$


Using this identity, we have

$$
\begin{aligned}
& (x) *(*(a) \wedge y)+*(y \wedge *(a \wedge x))-(\boldsymbol{x}, \boldsymbol{y}) \boldsymbol{a}, \boldsymbol{b}) \\
& \quad=(*(\boldsymbol{b}) \wedge \boldsymbol{x}, *(\boldsymbol{a}) \wedge \boldsymbol{y})+(\boldsymbol{x} \wedge \boldsymbol{a}, \boldsymbol{y} \wedge \boldsymbol{b})-(\boldsymbol{x}, \boldsymbol{y})(\boldsymbol{a}, \boldsymbol{b})=0 \\
& (*(\boldsymbol{a} \wedge *(\boldsymbol{b} \wedge \boldsymbol{x}))-*(*(\boldsymbol{b}) \wedge *(*(\boldsymbol{a}) \wedge \boldsymbol{x}))-(\boldsymbol{a}, \boldsymbol{b}) \boldsymbol{x}, \boldsymbol{y}) \\
& \quad=(\boldsymbol{x} \wedge \boldsymbol{b}, \boldsymbol{y} \wedge \boldsymbol{a})+(*(\boldsymbol{a}) \wedge \boldsymbol{x}, *(\boldsymbol{b}) \wedge \boldsymbol{y})-(\boldsymbol{x}, \boldsymbol{y})(\boldsymbol{a}, \boldsymbol{b})=0 .
\end{aligned}
$$

(6): Since

$$
\begin{aligned}
((x \times y) z, v) & =-(x \wedge z, y \wedge v)+4 / 5(x, y)(z, v) \\
& =(y, z)(x, v)-1 / 5(x, y)(z, v)
\end{aligned}
$$

we have

$$
(x \times y) z=(y, z) x-1 / 5(x, y) z .
$$

For $v, w \in \bigwedge^{1}\left(C^{5}\right)=C^{5}$, we have

$$
\begin{aligned}
& ((a \times *(b \wedge \boldsymbol{x})) v, w) \\
& =(v \wedge a, w \wedge *(b \wedge x))-3 / 5(*(a \wedge b), x)(v, w) \\
& =(v, w)(a, *(b \wedge x))-\left(a_{1}, w\right)\left(w \wedge a_{2}, *(b \wedge x)\right) \\
& \quad+\left(a_{2}, w\right)\left(w \wedge a_{1}, *(b \wedge x)\right)-3 / 5(*(a \wedge b), x)(v, w) \\
& =2 / 5(*(a \wedge b), x)(v, w)-\left(a_{1}, w\right)\left(b \wedge x \wedge w \wedge a_{2}, e_{1} \wedge \cdots \wedge e_{5}\right) \\
& \quad+\left(a_{2}, w\right)\left(b \wedge x \wedge w \wedge a_{1}, e_{1} \wedge \cdots \wedge e_{5}\right) \\
& ((b \times *(a \wedge x)) v, w) \\
& =(x \wedge a, w \wedge *(b \wedge v))-3 / 5(*(a \wedge b), x)(v, w) \\
& =(x, w)(*(a \wedge b), v)+\left(a_{1}, w\right)\left(b \wedge x \wedge w \wedge a_{2}, e_{1} \wedge \cdots \wedge e_{5}\right) \\
& \quad-\left(a_{2}, w\right)\left(b \wedge x \wedge w \wedge a_{1}, e_{1} \wedge \cdots \wedge e_{5}\right)-3 / 5(*(a \wedge b), x)(v, w)
\end{aligned}
$$

Using (10), we have

$$
\begin{aligned}
(\boldsymbol{a} \times *(\boldsymbol{b} \wedge \boldsymbol{x})) \boldsymbol{v}+(\boldsymbol{b} \times *(\boldsymbol{a} \wedge \boldsymbol{x})) v & =(\boldsymbol{x}, \boldsymbol{w}) *(\boldsymbol{a} \wedge \boldsymbol{b})-1 / 5(*(\boldsymbol{a} \wedge \boldsymbol{b}), \boldsymbol{x}) \boldsymbol{v} \\
& =(\boldsymbol{x} \times *(\boldsymbol{a} \wedge \boldsymbol{b})) v
\end{aligned}
$$


(7): We have

$$
\begin{aligned}
& ((a \times(x \wedge y)) v, w)=(x \wedge y \wedge w, v \wedge a)-3 / 5(a, x \wedge y)(v, w) \\
& =(x, v)(y \wedge w, a)-(y, v)(x \wedge w, a)+2 / 5(a, x \wedge y)(v, w) \\
& =(x, v)(*(*(a) \wedge y), w)-(y, v)(*(*(a) \wedge x), w)+2 / 5(a, x \wedge y)(v, w)
\end{aligned}
$$

On the other hand, using (10), we have

$$
\begin{aligned}
(*(*(a) \wedge \boldsymbol{x}) \times \boldsymbol{y}) \boldsymbol{v} & =(\boldsymbol{y}, \boldsymbol{v}) *(*(\boldsymbol{a}) \wedge \boldsymbol{x})-1 / 5(*(*(\boldsymbol{a}) \wedge \boldsymbol{x}), \boldsymbol{v}) \boldsymbol{y} \\
& =(\boldsymbol{y}, \boldsymbol{v}) *(*(\boldsymbol{a}) \wedge \boldsymbol{x})-1 / 5(\boldsymbol{a}, \boldsymbol{x} \wedge \boldsymbol{v}) y, \\
-(*(*(\boldsymbol{a}) \wedge \boldsymbol{x}) \times \boldsymbol{y}) \boldsymbol{v} & =-(\boldsymbol{x}, \boldsymbol{v}) *(*(\boldsymbol{a}) \wedge \boldsymbol{y})-1 / 5(\boldsymbol{a}, \boldsymbol{x} \wedge \boldsymbol{v}) y .
\end{aligned}
$$

Hence (7) has been proved. (8): Let us put $a=a_{1} \wedge a_{2}$ and $c=c_{1} \wedge c_{2}$. Since

$$
\begin{aligned}
((\boldsymbol{a} \times \boldsymbol{b}) \boldsymbol{v}, \boldsymbol{w}) & =(\boldsymbol{a} \wedge \boldsymbol{v}, \boldsymbol{b} \wedge \boldsymbol{w})-3 / 5(\boldsymbol{a}, \boldsymbol{b})(\boldsymbol{v}, \boldsymbol{w}) \\
& =-\left(a_{1}, \boldsymbol{w}\right)\left(\boldsymbol{x} \wedge \boldsymbol{a}_{2}, \boldsymbol{b}\right)+\left(a_{2}, \boldsymbol{w}\right)\left(\boldsymbol{x} \wedge \boldsymbol{a}_{1}, \boldsymbol{b}\right)+2 / 5(\boldsymbol{a}, \boldsymbol{b})(\boldsymbol{v}, \boldsymbol{w})
\end{aligned}
$$

we have

$$
\begin{aligned}
(a \times b) c= & -\left(a_{1} \wedge c_{1}, b\right) a_{2} \wedge c_{2}+\left(a_{1} \wedge c_{2}, b\right) a_{2} \wedge c_{1} \\
& +\left(a_{2} \wedge c_{1}, b\right) a_{1} \wedge c_{2}-\left(a_{2} \wedge c_{2}, b\right) a_{1} \wedge c_{1}+4 / 5(a, b) c
\end{aligned}
$$

On the other hand, for $d \in \bigwedge^{2}\left(C^{5}\right)$, we have

$$
\begin{aligned}
& (*(*(a \wedge \boldsymbol{c}) \wedge \boldsymbol{b}), \boldsymbol{d}) \\
& =(\boldsymbol{a} \wedge \boldsymbol{c}, \boldsymbol{b} \wedge \boldsymbol{d}) \\
& =(\boldsymbol{a}, \boldsymbol{b})(\boldsymbol{c}, \boldsymbol{d})-\left(\boldsymbol{a}_{1} \wedge c_{1}, \boldsymbol{b}\right)\left(a_{2} \wedge c_{2}, \boldsymbol{d}\right)+\left(a_{1} \wedge c_{2}, \boldsymbol{b}\right)\left(a_{2} \wedge c_{1}, \boldsymbol{d}\right) \\
& \quad+\left(a_{2} \wedge c_{1}, \boldsymbol{b}\right)\left(a_{1} \wedge c_{2}, \boldsymbol{d}\right)-\left(a_{2} \wedge c_{2}, \boldsymbol{b}\right)\left(a_{1} \wedge c_{1}, d\right)+(\boldsymbol{c}, \boldsymbol{b})(\boldsymbol{a}, \boldsymbol{d})
\end{aligned}
$$

Hence (8) has been proved. (9): Using (10), we have

$$
(x \times y) a=\left(y, a_{1}\right) x \wedge a_{2}-\left(y, a_{2}\right) x \wedge a_{1}-2 / 5(x, y) a .
$$

On the other hand, we have

$$
\begin{aligned}
(-*(y \wedge *(x \wedge a)), b) & =-(x \wedge a, y \wedge b) \\
& =\left(y, a_{1}\right)\left(x \wedge a_{2}, b\right)-\left(y, a_{2}\right)\left(x \wedge a_{1}, b\right)-(x, y)(a, b)
\end{aligned}
$$

Hence, (9) has proved. 
From Lemmas 1.1, 1.2 and 4.1, we can prove that $I$ becomes a graded (i.e., $\left[\mathfrak{l}_{k}, \mathfrak{l}_{l}\right] \subset \mathfrak{I}_{m}$ where $\left.m \equiv k+l \bmod 5\right)$ Lie algebra. Furthermore we have the following:

THEOREM 4.2. The Lie algebra I is a complex simple Lie algebra of type $E_{8}$.

Proof. Let $a$ be a non-zero ideal of $I$ and let us put

$$
\begin{aligned}
\mathfrak{I}_{01} & =\left\{(X, 0) \in I_{0} \mid X \in \mathfrak{s l}(5, C)\right\} \cong \mathfrak{s l}(5, C), \\
\mathfrak{I}_{02} & =\left\{(0, Y) \in I_{0} \mid Y \in \mathfrak{s l}(5, C)\right\} \cong \mathfrak{s l}(5, C), \\
\mathfrak{q} & =I_{1} \oplus \mathfrak{I}_{2} \oplus \mathfrak{I}_{3} \oplus \mathfrak{I}_{4} .
\end{aligned}
$$

There are three cases to be considered: (a) $\mathfrak{I}_{01} \cap \mathfrak{a}=\{0\}, \mathfrak{I}_{02} \cap \mathfrak{a}=\{0\}$ and $\mathfrak{q} \cap \mathfrak{a}=\{0\}$, (b) $\mathfrak{I}_{01} \cap \mathfrak{a} \neq\{0\}$ or $\mathfrak{I}_{02} \cap \mathfrak{a} \neq\{0\}$, (c) $\mathfrak{q} \cap \mathfrak{a} \neq\{0\}$.

Case (a): Let $p_{i}: \mathfrak{l} \rightarrow \mathfrak{I}_{0 i}(i=i, 2)$ denote the projection. If $p_{1}(\mathfrak{a})=\{0\}$ and $p_{2}(\mathfrak{a})=\{0\}$, then $\mathfrak{a}$ is contained in $\mathfrak{q}$, which contradicts to $\mathfrak{q} \cap \mathfrak{a}=\{0\}$. Hence, without loss of generality, we many assume $p_{1}(\mathfrak{a})=\mathfrak{I}_{01}$, because $\mathfrak{I}_{01}$ is a simple Lie algebra. For $X=\sum_{i=1}^{4} E_{i i}-4 E_{55} \in \mathfrak{s l}(5, C)$, there exists $\left(Y, \alpha_{1}, \alpha_{2}, \alpha_{3}, \alpha_{4}\right) \in$ $\mathfrak{I}_{02} \oplus \mathfrak{q}$ such that $\left(X, Y, \alpha_{1}, \alpha_{2}, \alpha_{3}, \alpha_{4}\right) \in \mathfrak{a}$. Since

$$
\begin{aligned}
& {\left[(X, 0),\left(X, Y, \alpha_{1}, \alpha_{2}, \alpha_{3}, \alpha_{4}\right)\right]} \\
& =\left(0,0,\left[X, \alpha_{1}\right],\left[X, \alpha_{2}\right],\left[X, \alpha_{3}\right],\left[X, \alpha_{4}\right]\right) \in \mathfrak{q} \cap \mathfrak{a}=\{0\}
\end{aligned}
$$

we have $\left[X, \alpha_{i}\right]=0(i=1,2,3,4)$. Since any eigenvalue of $\operatorname{ad} X$ is not 0 , we have $\alpha_{i}=0$. Then we have $(X, Y) \in \mathfrak{I}_{0} \cap \mathfrak{a}$. Since

$$
\left[(X, Y),\left(E_{45}, 0\right)\right]=\left(5 E_{45}, 0\right) \in \mathfrak{I}_{01} \cap \mathfrak{a}
$$

we have $I_{01} \cap \mathfrak{a} \neq\{0\}$. This is contradiction.

Case (b): We may assume $\mathfrak{I}_{01} \cap \mathfrak{a} \neq\{0\}$. Since $\mathfrak{I}_{01}$ is simple, we have $\mathfrak{I}_{01} \subset \mathfrak{a}$. Since $\left[\mathfrak{I}_{01}, \mathfrak{I}_{i}\right]=\mathfrak{I}_{i}(i \geq 1)$, we have $\mathfrak{q} \subset \mathfrak{a}$. Since

$$
\begin{aligned}
\mathfrak{a} \supset\left[\mathfrak{I}_{1}, \mathfrak{I}_{4}\right] & \ni\left[e_{1} \otimes\left(e_{1} \wedge e_{2}\right), e_{1} \otimes\left(e_{1} \wedge e_{3}\right)\right] \\
& =\left(0,-E_{23}\right)
\end{aligned}
$$

we have $I_{02} \cap \mathfrak{a} \neq\{0\}$. It follows that $I_{02} \subset \mathfrak{a}$. Hence we have $\mathfrak{a}=\mathfrak{I}$.

Case (c): Let $R=\left(\alpha_{1}, \alpha_{2}, \alpha_{3}, \alpha_{4}\right)\left(\alpha_{i} \in I_{i}\right)$ be a non-zero element of $\mathfrak{q} \cap \mathfrak{a}$. In case $\alpha_{1} \neq 0$, we put $\alpha_{1}=\sum_{i, j<k} \alpha_{i j k} e_{i} \otimes\left(e_{j} \wedge e_{k}\right)$. Without loss of generality, we may assume $\alpha_{112}=1$. Putting $S_{i j k l}=\left(E_{i i}-E_{j j}, E_{k k}-E_{l l}\right) \in \mathfrak{I}_{0}$ and $T=$ 
$e_{2} \otimes e_{1} \wedge e_{2} \in I_{4}$, we have

$$
\operatorname{ad}(T) \operatorname{ad}\left(S_{1523}\right) \operatorname{ad}\left(S_{1415}\right) \operatorname{ad}\left(S_{1314}\right) \operatorname{ad}\left(S_{1213}\right) R=\left(-E_{12}, 0\right) \in I_{01} \cap \mathfrak{a} .
$$

Then we can reduce this case to case (b). In case $\alpha_{i} \neq 0(i=2,3,4)$, we can similarly reduce to case (b).

Thus the simplicity of $I$ has been proved. On the other hand, since the dimension of $\mathrm{I}$ is clearly 248 , we see that $\mathrm{I}$ is a Lie algebra of type $E_{8}$.

Let us define a conjugate linear transformation $\gamma$ and an inner product $\left\langle R_{1}, R_{2}\right\rangle$ on I as follows:

$\gamma(X, Y, \boldsymbol{x} \otimes \boldsymbol{a}, \boldsymbol{b} \otimes \boldsymbol{y}, \boldsymbol{c} \otimes \boldsymbol{z}, \boldsymbol{w} \otimes \boldsymbol{d})=\left(-{ }^{t} \bar{X},-{ }^{t} \bar{Y}, \overline{\boldsymbol{w}} \otimes \overline{\boldsymbol{d}}, \overline{\boldsymbol{c}} \otimes \overline{\boldsymbol{z}}, \overline{\boldsymbol{b}} \otimes \overline{\boldsymbol{y}}, \overline{\boldsymbol{x}} \otimes \overline{\boldsymbol{a}}\right)$,

$$
\left\langle R_{1}, R_{2}\right\rangle=-B_{1}\left(R_{1}, \gamma R_{2}\right) \text {. }
$$

As in $\S 2$, we obtain

$$
\begin{aligned}
B_{\mathrm{I}}\left(R_{1}, R_{2}\right)= & 60 \operatorname{tr} X_{1} X_{2}+60 \operatorname{tr} Y_{1} Y_{2}-60\left(x_{1}, w_{2}\right)\left(a_{1}, d_{2}\right) \\
& -60\left(x_{2}, w_{1}\right)\left(a_{2}, d_{1}\right)-60\left(y_{1}, z_{2}\right)\left(b_{1}, c_{2}\right)-60\left(y_{2}, z_{1}\right)\left(b_{2}, c_{1}\right), \\
\left\langle R_{1}, R_{2}\right\rangle= & 60 \operatorname{tr} X_{1}^{t} \bar{X}_{2}+60 \operatorname{tr} Y_{1}^{t} \bar{Y}_{2}+60\left(x_{1}, \bar{x}_{2}\right)\left(a_{1}, \bar{a}_{2}\right)+60\left(y_{1}, \bar{y}_{2}\right)\left(b_{1}, \bar{b}_{2}\right) \\
& +60\left(z_{1}, \bar{z}_{2}\right)\left(c_{1}, \bar{c}_{2}\right)+60\left(w_{1}, \bar{w}_{2}\right)\left(d_{1}, \bar{d}_{2}\right) .
\end{aligned}
$$

Thus $\left\langle R_{1}, R_{2}\right\rangle$ is a positive definite Hermitian inner product on I. Using Lemma 1.2 (3) and (4), we see that $\gamma$ holds the Lie bracket. Then it is clear that $I^{\gamma}=$ $\{R \in \mathfrak{I} \mid \gamma(R)=R\}$ is the compact real form of $\mathfrak{l}$. Furthermore, from Proposition 1.4 , we see that the group

$$
E_{8}=\left\{\alpha \in \operatorname{Aut}(\mathrm{I}) \mid\left\langle\alpha R_{1}, \alpha R_{2}\right\rangle=\left\langle R_{1}, R_{2}\right\rangle\right\}
$$

is a simply connected compact simple Lie group of type $E_{8}$.

Put $\eta=\exp (2 \pi i / 5) \in C$ and define a transformation $\imath: \mathfrak{I} \rightarrow \mathfrak{I}$ as follows:

$$
l\left(X, Y, \alpha_{1}, \alpha_{2}, \alpha_{3}, \alpha_{4}\right)=\left(X, Y, \eta \alpha_{1}, \eta^{2} \alpha_{2}, \eta^{3} \alpha_{3}, \eta^{4} \alpha_{4}\right) \text {. }
$$

It is clear that $l \in E_{8}$ and $l^{5}=1$.

THEOREM 4.3. The subgroup $\left(E_{8}\right)^{l}$ of $E_{8}$ is isomorphic to the group $(S U(5) \times S U(5)) / Z_{5}$.

Proof. For any $A \in S U(5)$, we define a linear transformation $\psi_{1}(A)$ of I by

$$
\begin{aligned}
& \psi_{1}(A)(X, Y, \boldsymbol{x} \otimes \boldsymbol{a}, \boldsymbol{b} \otimes \boldsymbol{y}, \boldsymbol{c} \otimes \boldsymbol{z}, \boldsymbol{w} \otimes \boldsymbol{d}) \\
& =\left(\operatorname{Ad}(A) X, Y,(A \boldsymbol{x}) \otimes \boldsymbol{a},(A \boldsymbol{b}) \otimes \boldsymbol{y},\left({ }^{t} A^{-1} \boldsymbol{c}\right) \otimes z,\left({ }^{t} A^{-1} \boldsymbol{w}\right) \otimes d\right) .
\end{aligned}
$$


Obviously the map $\psi_{1}: S U(5) \rightarrow G L(\mathfrak{l})$ is a homomorphism. For any $Z \in \mathfrak{s u}(5)$, we have $(Z, 0) \in \mathrm{I}_{0}$ and

$$
\begin{aligned}
& \quad \exp (\operatorname{ad}(Z, 0))(X, Y, \boldsymbol{x} \otimes \boldsymbol{a}, \boldsymbol{b} \otimes \boldsymbol{y}, \boldsymbol{c} \otimes \boldsymbol{z}, \boldsymbol{w} \otimes \boldsymbol{d}) \\
& =(\exp (\operatorname{ad}(Z)) X, Y,((\exp Z) \boldsymbol{x}) \otimes \boldsymbol{a}, \\
& \left.\quad((\exp Z) \boldsymbol{b}) \otimes \boldsymbol{y},\left(\left(\exp \left(-{ }^{t} Z\right)\right) \boldsymbol{c}\right) \otimes \boldsymbol{z},\left(\left(\exp \left(-{ }^{t} Z\right)\right) \boldsymbol{w}\right) \otimes \boldsymbol{d}\right) \\
& =(\operatorname{Ad}(\exp Z) X, Y,((\exp Z) \boldsymbol{x}) \otimes \boldsymbol{a}, \\
& \left.\quad((\exp Z) \boldsymbol{b}) \otimes \boldsymbol{y},\left({ }^{t}(\exp Z)^{-1} \boldsymbol{c}\right) \otimes \boldsymbol{z},\left({ }^{t}(\exp Z)^{-1} \boldsymbol{w}\right) \otimes \boldsymbol{d}\right) \\
& =\psi_{1}(\exp Z)(X, Y, \boldsymbol{x} \otimes \boldsymbol{a}, \boldsymbol{b} \otimes \boldsymbol{y}, \boldsymbol{c} \otimes \boldsymbol{z}, \boldsymbol{w} \otimes \boldsymbol{d}) .
\end{aligned}
$$

It follows that $\psi_{1}(A)$ is an automorphism of I. Using Lemma 1.1, we have

$$
\left\langle\psi_{1}(A) R_{1}, \psi_{1}(A) R_{2}\right\rangle=\left\langle R_{1}, R_{2}\right\rangle
$$

Hence $\psi_{1}(A) \in E_{8}$. Similarly for any $B \in S U(5)$, we define a linear transformation $\psi_{2}(B)$ of I by

$$
\begin{aligned}
& \psi_{2}(B)(X, Y, \boldsymbol{x} \otimes \boldsymbol{a}, \boldsymbol{b} \otimes \boldsymbol{y}, \boldsymbol{c} \otimes \boldsymbol{z}, \boldsymbol{w} \otimes \boldsymbol{d}) \\
& =\left(X, \operatorname{Ad}(B) Y, \boldsymbol{x} \otimes(B \boldsymbol{a}), \boldsymbol{b} \otimes\left({ }^{t} B^{-1} \boldsymbol{y}\right), \boldsymbol{c} \otimes(B z), \boldsymbol{w} \otimes\left({ }^{t} B^{-1} \boldsymbol{d}\right)\right) .
\end{aligned}
$$

It is clear that the map $\psi_{2}: S U(5) \rightarrow G L(\mathrm{I})$ is a homomorphism, $\psi_{2}(B) \in E_{8}$ and $\psi_{1}(A) \psi_{2}(B)=\psi_{2}(B) \psi_{1}(A)$.

Furthermore we define a map $\varphi: S U(5) \times S U(5) \rightarrow\left(E_{8}\right)^{l}$ by

$$
\varphi(A, B)=\psi_{1}(A) \psi_{2}(B)
$$

Since $\imath=\psi_{1}(\eta I)$, we have $\imath \varphi(A, B)=\varphi(A, B) l$. Thus the map $\varphi$ is well-defined. Obviously $\varphi$ is a homomorphism. Also we can prove that $\varphi$ is surjective as in proof of Theorem 2.3.

At last, we shall show that $\operatorname{Ker} \varphi=\left\{\left(\eta^{m} I, \eta^{n} I\right) \mid m+2 n \equiv 0 \bmod 5\right\}$. For $(A, B) \in \operatorname{Ker} \varphi$, we have $A=\eta^{m} I$ and $B=\eta^{n} I(m, n \in Z)$. Since

$$
\begin{aligned}
\left(X, Y, \alpha_{1}, \alpha_{2}, \alpha_{3}, \alpha_{4}\right) & =\varphi\left(\eta^{m} I, \eta^{n} I\right)\left(X, Y, \alpha_{1}, \alpha_{2}, \alpha_{3}, \alpha_{4}\right) \\
& =\left(X, Y, \eta^{m+2 n} \alpha_{1}, \eta^{2 m-n} \alpha_{2}, \eta^{-2 m-n} \alpha_{3}, \eta^{-m-2 n} \alpha_{4}\right) \\
& =\left(X, Y, \eta^{m+2 n} \alpha_{1}, \eta^{2(m+2 n)} \alpha_{2}, \eta^{-2(m+2 n)} \alpha_{3}, \eta^{-(m+2 n)} \alpha_{4}\right)
\end{aligned}
$$


we have that $m+2 n \equiv 0 \bmod 5$. Then we have

$$
\operatorname{Ker} \varphi=\left\{(I, I),\left(\eta I, \eta^{2} I\right),\left(\eta^{2} I, \eta^{4} I\right),\left(\eta^{3} I, \eta I\right),\left(\eta^{4} I, \eta^{3} I\right)\right\} \cong Z_{5} .
$$

Therefore $(S U(5) \times S U(5)) / Z_{5} \cong\left(E_{8}\right)^{l}$ has been proved.

This theorem means that $\left(E_{8}\right)^{l}$ is a subgroup of type $A_{4} \times A_{4}$.

\section{References}

[1] Borel, A. and de Siebenthal, J., Les sous-groupes fermés de rang maximum des groupes de Lie clos, Comment. Math. Helv. 23 (1949), 200-221

[2] Helgason, S., Differential geometry, Lie groupes, and symmetric spaces, Academic Press, 1978.

[3] Imai, T. and Yokota, I., Non-compact simple Lie group $E_{8(-24)}$ of type $E_{8}$, J. Fac. Sci. Shinshu Univ. 15 (1980), 53-76.

[4] Imai, T. and Yokota, I., Simply connected compact simple Lie group $E_{8(-248)}$ of type $E_{8}, J$. Math. Kyoto Univ. 21 (1981), 741-762.

[5] Jacobson, N., Lie Algebras, Wiley Interscience, 1962.

[6] Rasevskii, P. K., A theorem on the connectedness of a subgroup of a simply connected Lie group commuting with any of its automorphisms, Trans. Moscow Math. Soc. 30 (1974), 3-22.

[7] Tits, J., Tabellen zu den einfachen Lie Gruppen und ihren Darstellungen, Lecture Notes no. 40, Springer-Verlag, Heidelberg, 1967.

[8] Wolf, J. A. and Gray, A., Homogeneous spaces defined by Lie group automorphisms I, J. Diff. Geometry 2 (1968), 77-114.

[9] Yokota, I. and Yasukura, O., Non-compact simple Lie group $E_{8(8)}$, Tsukuba J. Math. 10 (1986), 331-349.

[10] Yokota, I., Realizations of involutive automorphisms $\sigma$ and $G^{\sigma}$ of exceptional linear Lie groups $G$, part I, $G=G_{2}, F_{4}$ and $E_{6}$, part II, $G=E_{7}$, part III, $G=E_{8}$, Tsukuba J. Math. 14 (1990), 185-223, 379-404; 15 (1991), 301-314.

[11] Yokota, I., Realization of automorphisms $\sigma$ of order 3 and $G^{\sigma}$ of compact exceptional Lie groups $G, I, G=G_{2}, F_{4}, E_{6}$., J. Fac. Sci. Shinshu Univ. 20 (1985), 131-144.

[12] Yokota, I., Ishihara, T. and Yasukura, O., Subgroup $\left((S U(3) \times S U(6)) / Z_{3}\right) \cdot Z_{2}$ of the simply connected simple Lie group $E_{7}$, J. Math. Kyoto Univ. 23 (1983), 715-737.

Department of Mathematical Sciences

Yokohama City University

22-2 Seto, Kanazawa-ku

Yokohama, 236 Japan 